remained associated with incident HSV-2 after adjusting for socio-demographic, social and biological variables.

Conclusion The high HSV-2 rates among AGYW particularly those engaging in high-risk sexual behaviours underscores the need for HSV-2 control interventions to slow its spread and other STIs in KwaZulu-Natal. However, further research is required for more targeted interventions due to the lack of association between sexual behaviours and HSV-2 incidence.

\section{P321 A REVIEW OF TYPE 2 DIABETES AND TRADITIONAL RISK FACTORS IN A LARGE HIV+ COHORT}

${ }^{1}$ Z O'neill, ${ }^{2} \mathrm{D}$ Williams* ${ }^{1,2} \mathrm{~A}$ Chakera. 'BSMS, Brighton, UK; ${ }^{2} B S U H$, Brighton, UK

10.1136/sextrans-2021-sti.376

Introduction As antiretroviral therapy (ART) has become more effective, life expectancy of HIV+ patients has increased to normal levels. Therefore, there is an increased risk of developing other age-associated chronic illnesses, including type 2 diabetes (T2DM). Some older ARTs and protease inhibitors may increase the risk of developing insulin resistance and diabetes. This study aimed to measure prevalence of diabetes within a large HIV+ cohort and describe potential risk factors for developing diabetes and factors predictive of a poor outcome. Methods A case note review of a 2390-person HIV+ cohort was conducted and people with diabetes were identified. Data on demographics, HIV duration, ART exposure, diabetic risk factors, and comorbidities were collected.

Results 77 patients within the HIV+ cohort had T2DM. 63 were male $(81.8 \%)$ and the median age was 59.0 (Interquartile range 54-67). Median time between HIV and DM diagnosis was 16.3 years (IQR 12.4-24.0). The median ART duration was 16.3 years, with a median of 14.5 years from antiretroviral commencement until diabetes diagnosis (IQR 9.8-21.9). 50 (64.9\%) had been exposed to protease inhibitors. $17(22.1 \%)$ had a nadir CD4 <200. Many had risk factors for diabetes, 34/74 (45.9\%) had BMI $>30$ and 22 (28.6\%) were from minority ethnic groups. Factors associated with diabetic complications included 27 (35.1\%) had hyperlipidaemia and $33(42.9 \%)$ had hypertension.

Conclusion This study shows a prevalence of $3.2 \%$ of T2DM within a large HIV+ cohort, similar to the prevalence in the UK adult population. Traditional risk factors were common, and it seems a long duration of HIV infection and long exposure to ARV therapy, particularly protease inhibitors may increase risk. Factors associated with a poor prognosis were frequent, suggesting more aggressive management may be required in HIV+ patients with T2DM. Further research into the outcomes of such patients is needed.

\section{P323 STI PREVENTION IN BLACK MEN WHO HAVE SEX WITH MEN TAKING PREP: CONCERNS, COGNIZANCE, CONDOM NORMS AND MORE}

${ }^{1} \mathrm{M}$ Clement*, ${ }^{1} \mathrm{~J}$ Beckford, ${ }^{2} \mathrm{~J}$ Siren, ${ }^{1} \mathrm{M}$ Adorno, ${ }^{3} \mathrm{~S}$ Legrand, ${ }^{1} \mathrm{M}$ Bennett, ${ }^{4} \mathrm{~A}$ Corneli. ${ }^{1} \mathrm{LSU}$ Health Sciences Center, New Orleans, USA; ${ }^{2}$ CrescentCare Federally Qualified Health Center, New Orleans, USA; ${ }^{3}$ Duke Global Health Institute, Durham, USA; ${ }^{4}$ Duke Department of Population Health Sciences, Durham, USA

10.1136/sextrans-2021-sti.377

Introduction Profound sexual health disparities exist for Black men who have sex with men (MSM) in the US South, including high prevalence of sexually transmitted infections (STIs). STI prevention strategies beyond condoms are needed for Black MSM taking PrEP.

Methods We conducted in-depth interviews with Black MSM taking PrEP. Informed by the Health Belief Model, we asked about participants' STI knowledge; perceived susceptibility, severity, and concerns; and perceived benefits of STI prevention. We also asked about support systems, condom use norms, and various STI prevention strategies, particularly peer support via an mHealth application. Interviews were audiorecorded and analyzed using directed content analysis.

Results We interviewed 20 Black MSM ages 18-35. Eight had an STI diagnosis within 12 months; most $(n=15)$ had been taking PrEP for more than one year; 15 identified as gay, 4 as bisexual, and 1 as queer; and 17 had insurance. Participants felt well-informed about STI symptoms, transmission, and treatment. Most had concern about an STI diagnosis, noting shame or disappointment. Potential long-term effects were described as concerning but not frequently considered. Almost all reported strong social support from family or friends. Participants described being less likely to use condoms with routine partners or those on PrEP, noting the norm 'had switched from condoms to PrEP.' Most were willing to test for STIs more frequently (e.g. monthly), but desired convenience. Most said they would deliver partner therapy and some desired STI education. Although some did not use social media, most felt app-based social support would be helpful.

Conclusion Black MSM on PrEP voiced concern about STIs but many noted condom use was no longer the norm. They were willing to engage in methods focused on preventing STIs on an individual- or population-level. Most had sources of social support but still desired app-based peer support from other MSM

\section{P324 IMPLEMENTATION OF ROUTINE HPV TESTING IN MEN}

A Stary*, D Gruenbart, J Mühlberger, K Schwarz. Outpatient's Centre for Diagnosis of ST/S, Vienna, Austria

\subsection{6/sextrans-2021-sti.378}

Objective Rising awareness of human papillomavirus (HPV) infection in both genders leads to an increasing demand for HPV testing in men. In a previous study, two different collection devices were compared according to their performance and failure rate (CerviBrush and FLOQSwabs ${ }^{\circledR}$ ). Since the quality of the specimens was significantly better when taken with FLOQSwabs ${ }^{\circledR}$ (COPAN), all samples in this study were collected with FLOQSwabs ${ }^{\circledR}$ only. Aim of this study was to further evaluate the performance of FLOQSwabs ${ }^{\circledR}$ in a larger cohort to confirm the results already generated.

Methods In total 632 samples collected using the FLOQSwabs ${ }^{\circledR}$ from the penile, perigenital, pharyngeal and anorectal area were tested for HPV at the Outpatient's Centre for Diagnosis of STIs by using the Greiner Bio-One PapilloCheck ${ }^{\circledR}$ genotyping assay. Results HPV prevalence was 49.7\%. The most frequent HPV hr types were $16,53,51,66,39,59$ and 31. The highest prevalence of HPV hr types was detected in penis (41.5\%), urethral orifice and glans penis (39.5\%), followed by mons pubis (31.25\%). The most prevalent HPV lr types were 6 $(16.8 \%)$ and 42 (6.6\%). The highest prevalence of HPV lr 6 was detected on the scrotum (60\%) and the penis shaft (54.5\%). 